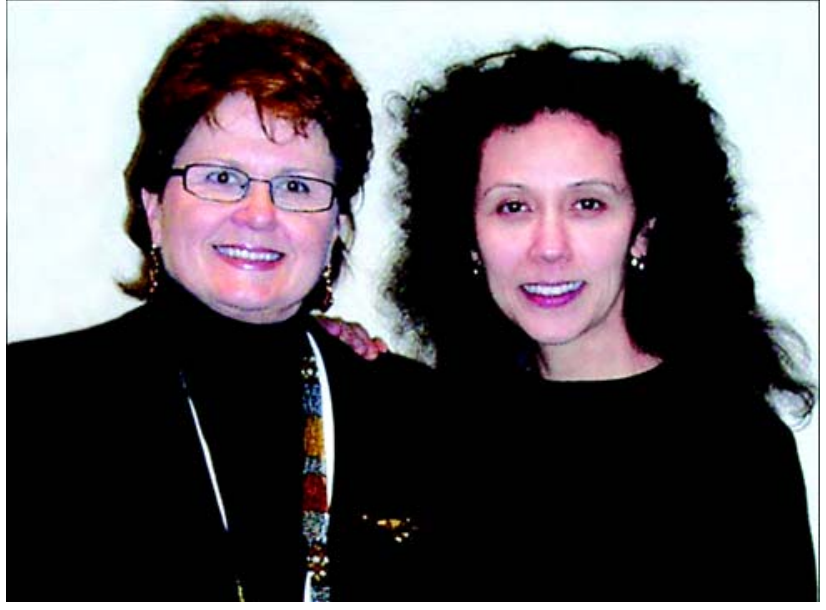

Leslie J. Gonzalez Rothi, PhD; Anna M. Barrett, MD

\section{Treatment innovation in behavioral rehabilitation of stroke: Removing limits on recovery}

Of the 5.4 million people in the United States who have had a stroke, half may have related motor and cognitive disabilities. Nearly one-quarter of stroke survivors need physical assistance in everyday life, and cognitive symptoms may have an equal or greater impact on function compared with physical limitations [1-3]. These numbers are frightening when we realize that health outcome studies in stroke may underrepresent the magnitude of the problem. Persons with disabilities who enter supervised or chronic-care settings may not be included in databases or research studies, and stroke survivors with cognitive disabilities may also fail to participate in research because of behavioral abnormalities, such as abnormal arousal and motivation, communication disorders, or the inability to coordinate activities and comply with study protocols. Although meticulous poststroke medical care is reducing stroke-related mortality in the United States, the number of survivors living with strokerelated disabilities may significantly increase as a result [4]. Thus, rehabilitation scientists are challenged to develop better and more effective treatments for cognitive and motor dysfunction so that people may return to satisfying life activities.

Neuroscience researchers routinely suggest that investigation of the mechanisms of stroke-related cognitive and motor deficits will inspire new treatments. However, basic research in poststroke deficits may only describe the correlates of disability rather than the degree of disability and may include subjects with subtle signs who would not ordinarily meet the clinical criteria for treatment. Thus, we as clinicians may have difficulty generalizing and applying the results of basic science experiments to the treatment of patient groups in a valid fashion. Most clinical researchers are aware of this limitation and regard mechanistic studies as a first step toward the development of treatment-valid research.

How to move from basic discovery toward large-scale, systematic clinical studies is not always as clear to the rehabilitation community [5]. That within-subject, exploratory, and experimental designs advance research questions and bridge the gap to randomized group studies may not be acknowledged. Scientists may criticize early phase clinical trial studies because they do not use randomized controls or meta-analysis, which are characteristic of phase III studies. However, the methodology of phase III research is neither appropriate nor desirable when a research question is innovative, being refined, and being explored for its feasibility and optimal setting.

An unfortunate truth is that even after quality randomized clinical trials of a treatment are available and the benefits indicated, problems occur when practitioners apply them to the treatment of individual patients. As practitioners apply a developed treatment, they may discover that the results do not 
generalize or do not apply to certain patients. Systematic investigation of these particular patients will give rise to data-refining clinically valid practice [6-7]. Such patients may belong to groups who were excluded or underrepresented in the formal trial but are frequently encountered in the aged population, such as patients with multiple chronic medical problems. Other understudied groups include women; members of ethnic, racial, and cultural minorities; rural dwellers; and the poor.

Averaged results from patients with diverse presentations of a clinical disorder in a large-scale clinical trial also may not represent the treatmentresponse spectrum. Patients with some theoretically defined subtypes of a clinical disorder may benefit, while others may experience adverse effects. Unless appropriate secondary analyses are performed, this disparity in response may go undetected and the trial reported as lacking any treatment benefit [8-9]. Such reexamination of "gold standard" results is desirable and, indeed, inevitable, as paradigm shifts in the field of cognitive rehabilitation help us redefine the brain-behavior mechanisms of the disorders that we treat.

We now know that people can learn new skills and knowledge well into late adulthood through experience and that this experience-dependent learning may be reflected in altered brain activity [10]. Can researchers and clinicians, in the context of the chronically injured adult brain, take advantage of this lifelong potential to help stroke survivors relearn lost behaviors? In this issue, the researchers report on their theory-driven investigations to determine specifically how we can bring the theory of neural recovery closer to the bedside of patients in need.

Three groups of studies are included in this issue. The first group includes reports on the potential effects of new treatments for poststroke aprosodia, apraxia of speech, spatial-cognitive disorders, and word retrieval in aphasia. In the second group, the researchers examine other factors in clinical trial evolution, including identification of predictors for outcome and refinement of assessment methods in poststroke swallowing disorders, dissociated response of speech and language functional components to treatment, and theoretical redirection and assessment of outcome in motor rehabilitation. The third group consists of two studies that examine alternate models of rehabilitative care delivery, specifically telerehabilitation and animal-assisted cognitive training.

In the past, clinicians and the lay public pervasively believed that stroke recovery was very limited. We are now entering an exciting period in poststroke care in which the time span and extent of continued improvement is extending incredibly. In another recent publication, we commented on the "new era of optimism” for cognitive recovery and poststroke cognitive rehabilitation [11]. An attitude of hope and expectation in stroke rehabilitation is likely to benefit survivors and families in and of itself. The articles in this issue demonstrate the powerful synergy of a scientific culture that seeks to dissolve barriers to continued progress for people with chronic poststroke deficits and to theoretically based, systematic research that respects the intermediate and extreme positions on the translational continuum.

\section{ACKNOWLEDGMENTS}

Mark V. Johnston, PhD, helped us identify literature on post-phase III individual-subject trialing.

This material was based on work supported by National Institutes of Health/National Institute of Neurological Disorders and Stroke, grant K02 NS047099, and the Henry H. Kessler Foundation (Dr. Barrett); and Department of Veterans Affairs Rehabilitation Research and Development Service (Dr. Gonzalez Rothi).

\section{Anna M. Barrett, MD; ${ }^{1-2}$ Leslie J. Gonzalez Rothi, $\mathbf{P h D}^{3-4 *}$}

${ }^{1}$ Kessler Medical Rehabilitation Research and Education Corporation, West Orange, NJ; ${ }^{2}$ Departments of Physical Medicine and Rehabilitation and Neurology/Neurosciences, University of Medicine and Dentistry of New Jersey, Newark, NJ; ${ }^{3}$ Brain Rehabilitation Research Center and Geriatric Research, Education, and Clinical Center, Malcom Randall Department of Veterans Affairs Medical Center, Gainesville, FL; ${ }^{4}$ Department of Neurology, University of Florida, Gainesville, FL

*Email: gonzalj@neurology.ufl.edu 


\section{REFERENCES}

1. American Heart Association Statistics Committee and Stroke Statistics Subcommittee. Heart disease and stroke statistics -2006 Update. Dallas (TX): American Heart Association; 2006.

2. Dobkin BH. Rehabilitation of specific neurologic disorders. Stroke: epidemiology. In: Dobkin BH, editor. The clinical science of neurologic rehabilitation. 2nd ed. New York (NY): Oxford University Press; 2003.

3. Barrett AM, Rothi LJG. Theoretical bases for neuropsychological interventions. In: Eslinger PJ, editor. Neuropsychological interventions: Clinical research and practice. New York (NY): Guilford Press; 2002. p. 16-37.

4. Thorvaldsen P, Kuulasmaa K, Rajakangas AM, Rastenyte D, Sarti C, Wilhelmsen L. Stroke trends in the WHO MONICA project. Stroke. 1997;28(3):500-506. [PMID: 9056602]

5. Rothi LJG. Cognitive rehabilitation: The role of theoretical rationales and respect for the maturational process needed for our evidence. J Head Trauma Rehabil. 2006;21(2): 194-97. [PMID: 16569992]
6. Ottenbacher KJ, Hinderer SR. Evidence-based practice: methods to evaluate individual patient improvement. Am J Phys Med Rehabil. 2001;80(10):786-96. [PMID: 11562563]

7. Guyatt G, Sackett D, Taylor DW, Chong J, Roberts R, Pugsley S. Determining optimal therapy-Randomized trials in individual patients. N Engl J Med. 1986;314(14): 889-92. [PMID: 2936958]

8. Barrett AM, Crucian GP, Schwartz RL, Heilman KM. Adverse effect of dopamine agonist therapy in a patient with motor-intentional neglect. Arch Phys Med Rehabil. 1999;80(5):600-603. [PMID: 10326927]

9. Berguer R. The evidence thing. Ann Vasc Surg. 2004; 18(3):265-70. [PMID: 15354626]

10. Small GW, Silverman DH, Siddarth P, Ercoli LM, Miller KJ, Lavretsky H, Wright BC, Bookheimer SY, Barrio JR, Phelps ME. Effects of a 14-day healthy longevity lifestyle program on cognition and brain function. Am J Geriatr Psychiatry. 2006;14(6):538-45. [PMID: 16731723]

11. Rothi LJG, Barrett AM. The changing view of neurorehabilitation: A new era of optimism [symposium editorial]. J Int Neuropsychol Soc. In press 2006.

DOI: 10.1682/JRRD.2006.08.0086 